\title{
Fatores de risco sociais, familiares e comportamentais para obesidade em adolescentes
}

\author{
Paulo Monteiro, ${ }^{1}$ Cesar Victora ${ }^{2}$ e Fernando Barros $^{3}$
}

Como citar Monteiro P, Victora C, Barros F. Fatores de risco sociais, familiares e comportamentais para obesidade em adolescentes. Rev Panam Salud Publica. 2004;16(4):250-8.

RESUMO Objetivo. Analisar fatores de risco sociais, familiares, comportamentais e psicológicos para obesidade na adolescência.

Método. Estudo de casos e controles aninhado a uma coorte de base populacional seguida desde o nascimento, em 1982. Os casos incluíram os adolescentes obesos conforme a definição da Organização Mundial da Saúde. Os controles foram constituídos por uma amostra aleatória da coorte, examinada em 1997 e 1998. Informações sobre fatores de risco foram obtidas dos arquivos da coorte, coletadas em diversas idades. A análise multivariada foi realizada por regressão logística.

Resultados. Os fatores de risco variaram conforme o sexo. Em meninos, a renda familiar maior que 1 salário-mínimo ao nascer aumentou o risco de obesidade em 6 vezes; o traço de ansiedade aumentou esse risco em 4 vezes. O risco aumentou em $10 \%$ para cada aumento unitário do índice de massa corporal materno pré-gestacional, em ambos os sexos. O peso materno atual, o fumo, a ingestão de gorduras, e o tempo despendido assistindo à televisão e em atividades físicas não se associaram com obesidade.

Conclusões. Os resultados indicam que é importante estratificar por sexo os dados de estudos sobre obesidade. Além disso, os fatores relativos a fases precoces da vida mostraram-se mais fortemente associados com obesidade em adolescentes do que os fatores medidos durante a adolescência. Entretanto, não se pode excluir a possibilidade de viés, por exemplo, em termos da informação fornecida pelos adolescentes acerca de dieta e exercício físico. Sempre que possível, o estudo dos fatores de risco contemporâneos para obesidade em adolescentes deve considerar o papel de fatores precoces na vida como prováveis fontes de confusão.

Palavras-chave Ansiedade, estudos de casos e controles, peso corporal.

1 Universidade Federal de Pelotas (UFPel), Departamento de Clínica Médica. Enviar correspondência para este autor no seguinte endereço: Avenida Duque de Caxias 250, Departamento de Medicina Social, Caixa Postal 464, CEP 96001-970, Pelotas, RS, Brasil. Fone/fax: +55-53-271-2442; e-mail: monteiro.po@terra.com.br

2 UFPel, Departamento de Medicina Social.

3 Organização Mundial da Saúde (OMS), Organização Pan-Americana da Saúde (OPAS), Centro Latino-Americano de Perinatologia e Desenvolvimento Humano.
A obesidade é a morbidade não transmissível que mais cresce em freqüência em todo o mundo (1), estando associada à ocorrência de diversas doenças e ao aumento da mortalidade, assim como a gastos crescentes com o tratamento de suas conseqüências diretas ou indiretas $(2,3)$. A obesidade vem apresentando rápido crescimento nos últimos anos mesmo em regiões onde sua freqüência é tradicionalmente baixa, como no Extremo Oriente (1). Aumentos na prevalência têm sido observados em diversas faixas etárias. Nos Estados Unidos da América, dados de 1999 mostram que o sobrepeso - definido como índice de massa corporal (IMC) entre 25,0 e 
$29,9 \mathrm{~kg} / \mathrm{m}^{2}$ - acomete $35 \%$ da população adulta (20 a 74 anos), e que a obesidade - definida como IMC $\geq 30$ $\mathrm{kg} / \mathrm{m}^{2}$ - acomete $27 \%$ da população da mesma faixa etária (4). No Brasil, a prevalência de obesidade (IMC $\geq 30$ $\mathrm{kg} / \mathrm{m}^{2}$ ) na população urbana foi estimada em cerca de $8,0 \%$ para os homens e $12,9 \%$ para as mulheres com 20 anos ou mais, em inquérito realizado em 1997 nas regiões Sudeste e Nordeste (5). Entretanto, dados do mesmo ano mostraram uma prevalência de obesidade de $25 \%$ em mulheres e $15 \%$ em homens na Cidade de Pelotas, no Sul do Brasil, para a população com idade entre 20 e 69 anos (6).

Os resultados de estudos entre adolescentes mostram um padrão semelhante. Nos Estados Unidos, mais de $26,5 \%$ dos adolescentes brancos apresentam-se em risco ou com sobrepeso (IMC $\geq$ percentil 85 para idade e sexo da população americana conforme pesquisa nacional de saúde e nutrição, a First National Health and Nutrition Examination Survey, ou NHANES I), sendo essa freqüência superior a $40 \%$ em americanos-mexicanos e negros não hispânicos (7). Nesse estudo, não houve diferenças importantes entre os sexos. Em estudo nacional realizado em 1989, a Região Sul do Brasil apresentou, também, a maior prevalência de sobrepeso (IMC $\geq$ ao percentil 85 da NHANES I) na adolescência: 13,9\% em mulheres e 7,2\% em homens (8). Usando o mesmo critério, um estudo realizado em Pelotas em 1998 relatou uma prevalência de sobrepeso de $20,9 \%$ em homens e $20,0 \%$ em mulheres com cerca de 15 anos (9). No mesmo estudo, 8,8\% dos adolescentes homens e 6,6\% das mulheres apresentaram obesidade, definida através de IMC e pregas cutâneas (10).

$\mathrm{Na}$ adolescência, a presença de obesidade tem sido associada a aumentos na pressão arterial e dislipidemias (11), a aumento na ocorrência de diabetes (12) e a distúrbios na esfera emocional (13). Além disso, a obesidade na adolescência determina maior ocorrência de obesidade e de doenças cardiovasculares na idade adulta (14). A ocorrência de obesidade na adolescência, por si só, está implicada em maior morbi-mortalidade na idade adulta (15). Em países desenvolvidos, a obesidade tem também sido associada a um menor grau de sucesso na vida profissional e a menores salários (16), embora em outros contextos (como no Oriente Médio e na Polinésia) a obesidade seja culturalmente admirada (1).

Da mesma forma, os estudos sobre os fatores de risco para obesidade na adolescência têm resultados diversos, às vezes conflitantes. Por exemplo, no Brasil, o estudo de 2000 mostrou que a freqüência de obesidade tendeu a crescer com a renda, mas essa relação se inverteu em mulheres mais abastadas das regiões urbanas (5). Outros fatores estudados incluem eventos de ocorrência precoce na vida, como o crescimento intra-uterino, a prematuridade, o peso materno, a amamentação e o crescimento precoce, além de fatores concorrentes à adolescência, como maturação sexual, nutrição, atividade física, estado emocional e tabagismo $(9,17-19)$.

Este estudo tem o objetivo de analisar fatores de risco para obesidade em adolescentes acompanhados desde o nascimento, em 1982, em uma cidade da Região Sul do Brasil, onde, como já descrito, se encontram as mais altas prevalências relatadas de obesidade nessa faixa etária no país. Em uma publicação anterior (9), abordamos os fatores de risco observados no início da vida e, no presente artigo, relatamos os fatores de risco medidos durante a adolescência. $\mathrm{O}$ conhecimento dos fatores ligados à obesidade é fundamental para permitir o desenvolvimento de ações voltadas ao controle desse crescente problema.

\section{MATERIAIS E MÉTODOS}

Foi utilizado um delineamento de casos e controles não emparelhados, aninhado a uma coorte acompanhada desde o nascimento. Em 1982, todos os 5914 nascimentos ocorridos em Pelotas, cidade de cerca de 300000 habitantes da Região Sul do Brasil, foram pesquisados. Desde então, essa coorte tem sido acompanhada em diversas ocasiões para avaliação, principalmente, de variáveis de morbidade, cresci- mento, desenvolvimento e nutrição. Em 1997 foram visitados 27\% dos setores censitários da Cidade de Pelotas, escolhidos sistematicamente, com o objetivo de identificar indivíduos nascidos em 1982 (20). Foram identificados 1076 adolescentes de 15 a 16 anos, que responderam a um questionário socioeconômico, demográfico, alimentar, comportamental e psicológico.

$\mathrm{O}$ peso e a altura foram medidos com balanças eletrônicas portáteis da UNICEF (Uniscale), com precisão de $0,1 \mathrm{~kg}$, e com antropômetros portáteis, com precisão de $0,1 \mathrm{~cm}$. Os adolescentes foram pesados com roupas leves, cujo peso estimado foi descontado do peso total. A altura foi tomada estando os adolescentes descalços e com a cabeça no plano de Frankfurt. Como nessa ocasião não foi possível medir as pregas cutâneas, realizou-se uma segunda visita a essa sub-amostra entre maio e outubro de 1998, quando foram buscados todos os indivíduos do quartil superior de IMC em 1997, e uma amostra aleatória simples de um terço dos indivíduos dos demais quartis de IMC. Maiores informações estão disponíveis em uma publicação anterior (21).

Os participantes foram visitados em seu domicílio por alunos do curso de medicina treinados especialmente para a coleta dos dados, que consistia em aplicar um questionário com questões demográficas, socioeconômicas, alimentares, comportamentais e sobre atividade física, além da realização de uma nova medida de peso e altura para determinação do IMC. Conforme recomendado pela Organização Mundial da Saúde (OMS) para diagnóstico de obesidade em adolescentes (10), nos indivíduos com IMC para idade e sexo acima do percentil 85 foram medidas dobras cutâneas subescapular e tricipital. As medidas foram padroniza-das, havendo os erros intra e interobservadores permanecido dentro dos padrões internacionais (22). Foram considerados obesos, ou casos, aqueles com IMC maior ou igual ao percentil 85 e com ambas as dobras cutâneas iguais ou superiores ao percentil 90 da mesma referência.

Informações sobre os seguintes fatores de risco foram obtidas através 
de questionário aplicado aos adolescentes e às suas mães: sexo; cor da pele (branca ou não branca, conforme autoclassificação do adolescente); renda familiar no último mês (dado coletado na visita de 1997); e estado nutricional da mãe em 1998 (IMC calculado a partir do peso e da altura medidos em 1998). Para a faixa etária estudada, esperavase que apenas cerca de 5\% dos meninos ainda não tivessem finalizado a fase de estirão do crescimento da adolescência. Investigaram-se, ainda, a idade na menarca em anos completos, o uso de tabaco (qualquer quantidade diária consumida foi considerada como uso), a presença de traço de ansiedade [48 ou mais pontos na escala de Spielberg, correspondente a um desvio-padrão (DP) ou mais acima da média local] $(23,24)$, o consumo de gordura (escore igual ou maior do que 22 no inventário de Block, que corresponde à ingestão, no último ano, de gordura igual ou superior à presente na dieta norteamericana típica) (25) e o tempo diário despendido assistindo à TV. Para estimar o gasto energético e padrões de atividade física, foram coletadas informações sobre o tempo, em minutos por dia, dias por semana e meses por ano, utilizado na realização de atividades domésticas e escolares, no lazer, no deslocamento e no trabalho, e calculado o gasto energético em equivalentes metabólicos (EM) (26). A variável utilizada nas análises foi obtida através da soma do tempo despendido em atividades físicas moderadas (que consomem entre 3 e 6 EM) e do dobro do tempo gasto em atividades intensas (que consomem mais de $6 \mathrm{EM}$ ), em 1 semana (27). A variável contínua foi recodificada em quartis.

As variáveis explanatórias correspondentes a fases anteriores da vida do adolescente foram obtidas através do banco de dados da coorte. Tais variáveis incluíram a renda familiar em 1982 e o IMC materno pré-gestacional (obtido através da mensuração da altura materna por ocasião do parto e do peso pré-gestacional obtido através da carteira de gestante ou do recordatório). Outras variáveis dos bancos de dados da coorte foram utilizadas apenas para controle de confundimento, quais sejam: a ordem de nascimento na prole, o peso ao nascer (em escores $Z$ ), e os escores $Z$ de peso para altura em 1984 (média de 20 meses de idade) e em 1986 (média de 43 meses de idade). Embora estivessem disponíveis os escores $\mathrm{Z}$ de peso para idade e altura para idade, optou-se pelo escore $Z$ de peso para altura por apresentar associação mais forte com obesidade na análise bivariada. Foi analisado, também, o crescimento rápido (acima de 0,67 escores $Z$ de peso para idade entre duas medidas) (9) entre os acompanhamentos realizados em 1982 e 1984 e 1984 e 1986 . Utilizou-se o escore Z contínuo pelo melhor ajuste do modelo multivariado. $\mathrm{O}$ mesmo tratamento foi dado às variáveis de IMC materno, que foram apresentadas em categorias na análise bivariada para facilitar a compreensão do leitor. Outra variável obtida no banco de dados da coorte foi o tempo de amamentação que, contudo, não se mostrou associado com obesidade $(P=0,70)$. Os resultados referentes a essas variáveis não são apresentados no presente artigo.

A análise de casos e controles constou da comparação dos adolescentes obesos com os demais adolescentes. Em função da forma como a amostra foi selecionada, houve uma sobrerepresentação dos controles oriundos do grupo acima do percentil 75 (P75) de IMC em 1997 ( $n=166)$, numa proporção de 3:1 em relação ao grupo abaixo desse percentil. Por esse motivo, e para equilibrar a amostra, esses controles foram ponderados à razão de 0,33:1 em relação aos controles pertencentes aos quartis inferiores de IMC em 1997. Da mesma forma, dois indivíduos classificados como obesos em 1998, e que pertenciam aos quartis inferiores ao P75 em 1997, foram ponderados numa razão de 3:1 em relação aos demais casos.

Das variáveis de exposição estudadas, aquela de menor prevalência nos indivíduos não obesos foi o traço de ansiedade $(14,1 \%)$, e a de maior prevalência foi a ingestão moderada de gorduras (72,5\%). Para ambas, a amostra permitiu que se detectasse uma razão de chances (odds ratio, OR) de 2,4 e 2,6 , respectivamente, com um poder de $80 \%$ e um nível de significância de $95 \%$. Para as demais variáveis estudadas a amostra permitiu que se detectassem valores de OR em torno de 2,0 com o mesmo poder e nível de significância.

Foram realizadas análises bivariadas para avaliação das associações brutas das variáveis explanatórias com obesidade. Também se utilizou essa estratégia de análise para determinar os possíveis fatores de confusão. Utilizamos para a análise multivariada um modelo de determinação hierarquizada (28) por níveis de causalidade em cinco níveis. Essa modelagem definiu a ordem de entrada das variáveis no modelo de regressão utilizado. Primeiro as variáveis do nível I foram ajustadas entre si. Após, entraram na regressão as variáveis do nível II, e assim sucessivamente. Foram mantidas na análise multivariada, para controle de confundimento, as variáveis que se associaram com o desfecho a um $P<0,20$ (29). Foram consideradas associações significativas com obesidade aquelas com $P<0,05$. Tanto as análises bivariadas quanto as multivariadas foram realizadas pelo método de regressão logística não-condicional, sendo a medida de efeito a OR. Todas as análises foram realizadas no pacote estatístico Stata 7.0.

Objetivando maximizar a qualidade das informações coletadas, os entrevistadores foram treinados para a aplicação dos questionários por meio de dramatizações, e as medidas antropométricas foram padronizadas. Um estudo piloto foi realizado para testar tanto os instrumentos quanto a coleta dos dados. Além disso, cerca de 5\% das entrevistas foram repetidas pelo supervisor do trabalho de campo. $\mathrm{O}$ banco de dados foi construído por dupla digitação, procedendo-se à análise de consistência dos dados.

Os comitês de ética das duas universidades envolvidas no estudo (Universidade Católica de Pelotas e Universidade Federal de Pelotas), os quais estão vinculados à Comissão Nacional de Ética em Pesquisa, aprovaram todas as fases do estudo. O consentimento informado verbal foi obtido junto aos responsáveis nos estudos rea- 
lizados entre 1982 e 1986, conforme a prática de consentimento da época. Nas fases mais recentes do estudo optou-se pelo registro, por escrito, do consentimento informado. A confidencialidade foi garantida em todas as fases do estudo, e os arquivos com os nomes das mães e dos componentes da coorte são mantidos separadamente e sob acesso restrito.

\section{RESULTADOS}

Em 1997 foram visitados 27\% do total de setores censitários. Nesses setores, os pesquisadores esperavam encontrar 27\% dos 5914 nascidos vivos em 1982, ou seja, 1597 indivíduos. Foram efetivamente localizados 1 076. A esse número devem ser acrescidos $27 \%$ dos indivíduos que morreram até 1997, ou seja, 70 indivíduos, para um total de 1597 indivíduos. Dessa forma, o percentual de indivíduos acompanhados até 1997 foi de $71,8 \%$. A descrição das perdas por grupo social está apresentada em outro trabalho (20). Devido ao fato de os indivíduos não localizados em diferentes visitas de acompanhamento não serem os mesmos, as freqüências de dados ignorados para algumas variáveis diferiram entre si, sendo que o IMC materno pré-gestacional foi a variável com maior percentual de dados ignorados, de 15,7\%.

Dos 528 indivíduos visitados em 1997 e escolhidos para serem revisitados em 1998, 25 (4,7\%) recusaram-se a participar ou não foram encontrados. Logo, 503 foram efetivamente entrevistados em seus domicílios. Desses, 10 adolescentes do sexo feminino foram retiradas da análise por estarem grávidas, e uma por falta de dados nos acompanhamentos iniciais da coorte. Portanto, foram efetivamente levados para a análise 492 indivíduos, sendo 242 homens e 250 mulheres.

A tabela 1 mostra o resultado da análise bruta para a associação entre a obesidade e as variáveis demográficas, socioeconômicas, biológicas, comportamentais e de crescimento e a atividade física. Não há diferença na ocorrência de obesidade em relação a cor da pele, mas a associação com sexo esteve no limiar da significância estatística $(P=0,062)$.

A obesidade associou-se positiva e linearmente com a renda familiar em 1982 , sendo quase 4 vezes maior nas famílias cuja renda era superior a 1 salário-mínimo. Já a associação com os quartis de renda em 1997 foi de menor magnitude, não havendo tendência linear, embora o teste de heterogeneidade tenha sido significativo $(P=$ $0,019)$. Optou-se por analisar a renda de 1997 em quartis, pois, quando foram empregadas as mesmas categorias de 1982, não houve casos de obesidade na categoria com renda de até 1 salário-mínimo.

A obesidade também se associou positiva e linearmente com o IMC materno antes da gestação, sendo 3,5 a 4 vezes maior para adolescentes cujas mães apresentavam sobrepeso e obesidade, respectivamente. $\mathrm{O}$ mesmo tipo de associação ocorreu em relação ao IMC materno em 1998, embora com maior magnitude relacionada à obesidade materna. Não houve associação entre a mudança de IMC materno entre 1982 e 1997 e a presença de obesidade no adolescente.

O risco de obesidade foi $67 \%$ menor nas meninas cuja menarca ocorreu após os 11 anos de idade. A menarca ocorreu aos 11,8 anos, em média, nos casos, e aos 12,5 anos nos controles $(P=0,002$, dado não apresentado). $\mathrm{O}$ risco de obesidade foi $74 \%$ maior entre os indivíduos com traço de ansiedade, embora a significância estatística tenha sido limítrofe $(P=0,071)$. Ainda, o risco de obesidade foi pelo menos a metade nos que assistiam à televisão por até 4 horas diárias, quando comparados com os que assistiam à televisão por 5 horas ou mais. $\mathrm{O}$ fumo, a ingestão de gorduras e o tempo despendido em atividades físicas não se associaram com um maior risco de obesidade nos adolescentes da coorte.

A tabela 2 mostra a análise multivariada para as associações com obesidade, incluindo análises estratificadas por sexo. As variáveis explanatórias associadas à obesidade com $P<0,20$ foram mantidas no modelo multivariado como possíveis fatores de con- fusão. A apresentação por níveis refere-se à forma de entrada das variáveis no modelo de análise por regressão logística.

A cor da pele não esteve associada, nem na análise bruta nem na ajustada, com a obesidade. A magnitude dos efeitos sobre a obesidade da renda familiar em 1982 e do IMC materno antes da gestação não se modificou após o ajuste, sendo a OR de obesidade 3 vezes maior naqueles cujas famílias ganhavam 2 ou mais salários. A análise estratificada, no entanto, mostrou que essa associação se manteve significativa apenas para o sexo masculino, com grande magnitude (cerca de 6 vezes mais risco de obesidade entre os meninos cujas famílias tinham renda de 2 ou mais salários).

A cada aumento unitário no IMC materno pré-gestacional correspondeu um aumento de cerca de $10 \%$ no risco de obesidade nos adolescentes, sendo que essa associação ocorreu com força e significância semelhantes em ambos os sexos.

Menarca após os 11 anos de idade, que se mostrou o fator de proteção para obesidade na análise bruta, perdeu sua significância na análise multivariada, sobretudo quando controlada para o IMC materno pré-gestacional.

A magnitude da associação positiva entre a renda familiar em 1997 e a obesidade não se modificou na análise ajustada, mas a precisão da OR foi seriamente afetada pela inclusão, no modelo, da renda em 1982, que apresenta um alto grau de colinearidade. A análise estratificada por sexo mostrou uma associação limítrofe $(P=0,11)$ para o sexo masculino. O IMC materno em 1998 não se mostrou associado com obesidade após o ajuste, tampouco após a estratificação por sexo.

O risco de obesidade foi 4 vezes maior entre os meninos classificados como tendo traço de ansiedade. Essa associação somente apareceu na análise multivariada estratificada por sexo. Por outro lado, o fumo e a ingestão de gorduras não se associaram com obesidade.

A análise ajustada para o risco relacionado ao tempo despendido assistindo à televisão não mostrou 
TABELA 1. Características dos adolescentes nascidos em 1982 e incluídos no estudo sobre os fatores de risco para obesidade, Pelotas (RS), Brasil, 1998

\begin{tabular}{|c|c|c|c|c|}
\hline Variáveis & $\begin{array}{c}\% \text { de casos } \\
(n=85)\end{array}$ & $\begin{array}{l}\text { \% de controles } \\
\quad(n=407)\end{array}$ & OR $(\operatorname{IC~} 95 \%)^{a}$ & $P^{\mathrm{b}}$ \\
\hline \multicolumn{5}{|l|}{ Sexo } \\
\hline Feminino & 40,0 & 53,1 & $0,63(0,38$ a 1,02$)$ & 0,062 \\
\hline \multicolumn{5}{|l|}{ Cor da pele } \\
\hline Branca & 74,1 & 70,8 & 1,0 & \\
\hline Não branca & 25,9 & 29,2 & $0,80(0,46$ a 1,38$)$ & 0,42 \\
\hline$>1,1 \mathrm{e} \leq 3$ & 50,6 & 47,0 & $3,54(1,33$ a 9,43$)$ & \\
\hline$>3$ & 43,5 & 36,9 & $3,71(1,38$ a 10,0$)$ & $0,04^{\mathrm{d}}$ \\
\hline \multicolumn{5}{|c|}{ Índice de massa corporale materno pré-gestacional $\left(\mathrm{kg} / \mathrm{m}^{2}\right)$} \\
\hline$<21,0$ & 16,7 & 32,7 & 1,0 & \\
\hline$\geq 21,0$ e $<23,0$ & 29,2 & 24,8 & $2,79(1,26$ a 6,15$)$ & \\
\hline$\geq 23,0$ e $<25,0$ & 16,7 & 15,5 & $2,37(0,98$ a 5,68$)$ & \\
\hline \multicolumn{5}{|c|}{ Renda familiar em 1997 (salários-mínimos) } \\
\hline$<2,7$ & 10,7 & 23,1 & 1,0 & \\
\hline$\geq 2,7$ e $\leq 5,0$ & 34,5 & 25,4 & $3,40(1,50$ a 7,70$)$ & \\
\hline$>5,0$ e $\leq 9,8$ & 25,0 & 25,4 & $2,26(0,98$ a 5,23$)$ & \\
\hline$>9,8$ & 29,8 & 26,1 & $2,87(1,25$ a 6,60$)$ & $0,019^{c}$ \\
\hline \multicolumn{5}{|c|}{ Índice de massa corporale materno em $1998\left(\mathrm{~kg} / \mathrm{m}^{2}\right)$} \\
\hline$<21,0$ & 2,4 & 6,6 & 1,0 & \\
\hline$\geq 21,0$ e $<23,0$ & 4,8 & 11,4 & $1,17(0,20$ a 6,92$)$ & \\
\hline$\geq 23,0$ e $<25,0$ & 19,3 & 15,6 & $4,33(0,90$ a 20,77$)$ & \\
\hline$\geq 25,0$ e $<30,0$ & 31,3 & 41,0 & $2,87(0,63$ a 13,04$)$ & \\
\hline$\geq 30$ & 42,2 & 25,4 & $5,86(1,31$ a 26,31$)$ & $0,001^{e}$ \\
\hline \multicolumn{5}{|l|}{ Idade na menarca (anos) $(n=248)$} \\
\hline$\leq 11$ & 47,1 & 27,6 & 1,0 & \\
\hline Normal & 25,9 & 28,3 & 1,0 & \\
\hline Elevada & 74,1 & 71,7 & $1,13(0,66$ a 1,96$)$ & 0,66 \\
\hline \multicolumn{5}{|l|}{ Horas de TV por dia } \\
\hline Até 1 & 7,1 & 10,8 & $0,45(0,17$ a 1,18$)$ & $0,053^{\mathrm{e}}$ \\
\hline 2 a 4 & 55,3 & 59,5 & $0,65(0,38$ a 1,11$)$ & \\
\hline 5 ou mais & 37,6 & 29,7 & 1,0 & \\
\hline \multicolumn{5}{|c|}{ Horas em atividades físicas moderadas e intensas } \\
\hline$\leq 2,68$ & 20,2 & 26,0 & 1,0 & \\
\hline$>2,68 \mathrm{a} \leq 4,40$ & 28,6 & 24,1 & $1,60(0,79$ a 3,24$)$ & \\
\hline$>4,40 \mathrm{a} \leq 7,40$ & 28,6 & 24,6 & $1,63(0,80$ a 3,30$)$ & \\
\hline$>7,40$ & 22,6 & 25,3 & $1,19(0,58$ a 2,45$)$ & $0,63^{\mathrm{e}}$ \\
\hline
\end{tabular}

a $\mathrm{OR}$ = Razão, entre casos e controles, da proporção de indivíduos expostos e não expostos à variável estudada.

${ }^{\mathrm{b}}$ Teste de $\chi^{2}$ de Pearson.

c Equivalente a US\$90,00 na época do estudo.

d Teste de $\chi^{2}$ para tendência linear.

e Índice de massa corporal = peso em $\mathrm{kg} \div$ altura em $\mathrm{m}^{2}$.

${ }^{\dagger}$ Média da variação $=4,3 \mathrm{~kg} / \mathrm{m}^{2} ;$ desvio-padrão $=3,96 \mathrm{~kg} / \mathrm{m}^{2}$.

9 Avaliado pelo Inventário de Ansiedade Traço-Estado (IDATE) (24).

${ }^{\mathrm{h}}$ Conforme inventário de Block (25) 
TABELA 2. Análise multivariada por níveis de causalidade, estratificada por sexo, para as associações entre diversas variáveis e o risco de obesidade em uma coorte de adolescentes nascidos em 1982, Pelotas (RS), Brasil 1998

\begin{tabular}{|c|c|c|c|c|}
\hline \multirow[b]{2}{*}{ Níveis $^{\mathrm{c}}$} & \multirow[b]{2}{*}{ Variáveis } & \multicolumn{3}{|c|}{ OR ajustada a,b (IC 95\%) } \\
\hline & & Toda a amostra $(n=492)$ & Homens $(n=242)$ & Mulheres $(n=250)$ \\
\hline \multirow[t]{3}{*}{ I } & Cor da pele & & & \\
\hline & Não branca & $0,89(0,51$ a 1,57$)$ & $0,90(0,43$ a 1,88$)$ & $0,85(0,36$ a 2,00$)$ \\
\hline & & $P=0,69$ & $P=0,78$ & $P=0,70$ \\
\hline \multirow[t]{3}{*}{ I } & Renda familiar em 1982 (salários-mínimos) e & & & \\
\hline & $>3$ & $3,62(1,33$ a 9,88$)$ & $6,48(1,51$ a 32,23$)$ & $1,60(0,43$ a 6,03$)$ \\
\hline & & $P=0,027$ & $P=0,011$ & $P=0,77$ \\
\hline \multirow[t]{2}{*}{ II } & Aumento unitário do índice de massa corporal materno & & & \\
\hline & & $\begin{array}{c}1,12(1,05 \text { a } 1,19)^{d} \\
P=0,001\end{array}$ & $\begin{array}{c}1,09(1,01 \text { a } 1,19)^{d} \\
P=0,026\end{array}$ & $\begin{array}{c}1,16(1,04 \text { a } 1,30)^{d} \\
P=0,008\end{array}$ \\
\hline \multirow{2}{*}{ III } & Renda familiar em 1997 (salários-mínimos) & & & \\
\hline & & $P=0,18$ & $P=0,11$ & $P=0,68$ \\
\hline \multirow[t]{2}{*}{ IV } & Aumento unitário do índice de massa corporal materno & & & \\
\hline & medido em 1998 & $\begin{array}{c}1,03(0,96 \text { a } 1,11)^{g} \\
P=0,41\end{array}$ & $\begin{array}{c}0,99(0,90 \text { a } 1,09)^{9} \\
P=0,86\end{array}$ & $\begin{array}{c}1,08(0,94 \text { a } 1,25)^{g, h} \\
P=0,27\end{array}$ \\
\hline \multirow[t]{3}{*}{ IV } & Idade na menarca (anos) & & & \\
\hline & $\leq 11$ & Não se aplica & Não se aplica & 1,09 \\
\hline & $>11$ & & & $\begin{array}{c}0,69(0,27 \text { a } 1,76) \\
P=0,43\end{array}$ \\
\hline \multirow[t]{4}{*}{ V } & Traço de ansiedade & & & \\
\hline & Não & $1,0^{\mathrm{i}}$ & $1,0^{\mathrm{i}}$ & $1,0^{\mathrm{h}, \mathrm{i}}$ \\
\hline & Sim & $1,93(0,86$ a 4,35$)$ & $3,98(1,06$ a 15,00$)$ & $2,00(0,63 a$ 6,33) \\
\hline & & $P=0,112$ & $P=0,041$ & $P=0,24$ \\
\hline V & Uso de tabaco & & & \\
\hline \multirow{3}{*}{$\mathrm{V}$} & 2 a 4 & $0,30(0,09$ a 1,03$)$ & $0,87(0,32$ a 2,40$)$ & $0,79(0,30$ a 2,10$)$ \\
\hline & 5 ou mais & $1,0^{i}$ & $1,0^{i}$ & $1,0^{\mathrm{h}, \mathrm{i}}$ \\
\hline & & $P=0,102$ & $P=0,53$ & $P=0,063$ \\
\hline \multirow[t]{6}{*}{ V } & Horas de atividade física moderada e intensa por dia & & & \\
\hline & $\leq 2,68$ & $1,0^{i}$ & $1,0^{\mathrm{i}}$ & $1,0^{\mathrm{h}, \mathrm{i}}$ \\
\hline & $>2,68 \mathrm{a} \leq 4,40$ & $1,46(0,59$ a 3,65$)$ & $0,71(0,17$ a 2,93$)$ & $2,49(0,70$ a 8,85$)$ \\
\hline & $>4,40 \mathrm{a} \leq 7,40$ & $1,81(0,69$ a 4,70$)$ & $1,81(0,44$ a 7,44$)$ & $1,04(0,27$ a 4,00$)$ \\
\hline & $>7,40$ & $1,44(0,59$ a 3,52$)$ & $1,20(0,36$ a 4,03$)$ & $1,29(0,24$ a 6,96$)$ \\
\hline & & $P=0,36$ & $P=0,38$ & $P=0,89$ \\
\hline
\end{tabular}

a Teste de Wald. Nas variáveis com mais de duas categorias, os valores indicam tendência linear.

${ }^{\mathrm{b}}$ Todas as variáveis ajustadas também para o sexo.

c Níveis hierárquicos de causalidade.

${ }^{\mathrm{d}}$ Ajustadas para variáveis do nível $\mathrm{l}$.

e Equivalente a US $\$ 90,00$ na época do estudo.

${ }^{\dagger}$ Ajustadas para variáveis dos níveis I e II, além de primogenitura, escore $Z$ de peso ao nascer, escore $Z$ de peso para comprimento em 1984 e escore $Z$ de peso para altura em 1986.

g Ajustadas para variáveis dos níveis I, II e III, além de primogenitura, escore $Z$ de peso ao nascer, escore $Z$ de peso para comprimento em 1984 e escore $Z$ de peso para altura em 1986.

${ }^{\mathrm{h}}$ No sexo feminino, as variáveis de níveis IV e $\mathrm{V}$ foram também ajustadas para a idade na menarca.

' Ajustadas para variáveis dos níveis I, II, III, IV e V, além de primogenitura, escore Z de peso ao nascer, escore Z de peso para comprimento em 1984 e escore $Z$ de peso para altura em 1986. 
associação na amostra global. A interpretação da análise estratificada por sexo foi prejudicada pelo número reduzido de observações.

\section{DISCUSSÃO}

Este estudo, por ser aninhado em uma coorte populacional, permite estudar exposições atuais e passadas, reduzindo o viés de recordatório, e permitindo avaliar o papel de mudanças temporais em exposições como renda familiar e IMC materno. A ocorrência de perdas de acompanhamento é um dos principais problemas dos estudos de coorte, pois pode comprometer a representatividade do grupo original. Neste estudo, após 16 anos de acompanhamento, cerca de $30 \%$ da coorte original não foram localizados. As perdas foram mais freqüentes no grupo de renda familiar mais baixa, onde $61 \%$ dos indivíduos foram localizados, em relação ao grupo de renda mais alta, onde esse percentual foi de $79 \%$ (20). No entanto, o modelo multivariado foi ajustado para renda familiar, reduzindo a possibilidade de viés. Também a presença de valores ignorados poderia comprometer a validade externa do estudo. Entretanto, a prevalência de obesidade em toda a amostra foi igual a $7,7 \%$ e, quando considerada a prevalência apenas naqueles com dados completos (70\% da amostra), a prevalência foi de 7,8\%. Também se deve considerar que a amostra reduzida na análise estratificada por sexo pode ter comprometido o poder estatístico do estudo.

Entre as 13 variáveis estudadas, a obesidade esteve associada significativamente, ou no limiar da significância, ao sexo, à renda familiar em 1982 e 1997, ao IMC materno pré-gestacional e ao IMC materno em 1998, à idade na menarca, à presença de traço de ansiedade e ao tempo gasto assistindo à televisão. $\mathrm{Na}$ análise multivariada para ambos os sexos, apenas a renda familiar em 1982 e o IMC pré-gestacional permaneceram associados à obesidade. Após estratificar por sexo, ainda na análise multivariada, observou-se que a renda em 1982, o IMC materno pré- gestacional e a ansiedade estavam associados com obesidade nos meninos, enquanto que o IMC pré-gestacional e o hábito de assistir à televisão foram fatores de risco para as meninas. Esses resultados serão discutidos a seguir.

A obesidade associou-se com maior renda entre os meninos, não havendo diferença entre as meninas. A renda atual apresentou associação limítrofe $(P=0,11)$, enquanto a renda ao nascer se mostrou um fator de risco mais importante $(P=0,011)$. Não se observaram diferenças conforme a cor da pele. Nos Estados Unidos, o sobrepeso é mais prevalente entre hispânicos e negros, e no sexo feminino é inversamente proporcional à renda (7), o que tem sido observado em outros países desenvolvidos $(30,31)$.

A associação entre IMC materno em 1998 e obesidade desapareceu na análise ajustada, enquanto a associação com IMC materno pré-gestacional se manteve, sugerindo que fatores genéticos ou ambientais em fases precoces da vida determinam a ocorrência de obesidade na adolescência, como mostrado em artigo anterior (9). A associação de sobrepeso e obesidade na adolescência com obesidade atual dos pais tem sido relatada $(32,33)$. No presente estudo, essa associação somente foi encontrada na análise bruta, pois ao ajustarmos o modelo para variáveis perinatais, especialmente para o peso materno pré-gestacional, a associação desapareceu. Analisou-se a variável em categorias, como na análise bruta, e como contínua, mas o resultado não se modificou. Nem mesmo a ocorrência de aumento do IMC materno em cerca de $4 \mathrm{~kg} / \mathrm{m}^{2}$ (mais de $1 \mathrm{DP}$ ) entre os acompanhamentos de 1982 a 1998 esteve associada à obesidade no adolescente, reforçando a inferência acima descrita.

A menarca antes dos 11 anos se associou com maior risco de obesidade na análise bruta. Um estudo brasileiro de 1998 encontrou uma associação inversa entre a menarca e a obesidade, após controle para variáveis medidas na adolescência, incluindo os fatores nutricionais, a atividade física, o peso materno e paterno e as horas despendidas diariamente assistindo à tele- visão (32). No presente estudo, a associação se manteve quando se procedeu ao mesmo tipo de análise multivariada para fatores medidos na adolescência (dados não mostrados), mas desapareceu quando ajustada para fatores de confusão perinatais (tabela 2). A inclusão, no modelo, do IMC materno pré-gestacional teve o maior efeito de confusão sobre a menarca. Ainda, apenas $7,1 \%$ das adolescentes tomavam anticoncepcionais orais, e nenhuma delas era obesa.

A associação entre o traço de ansiedade e a obesidade em meninos foi surpreendente. A literatura a respeito é controversa. Um estudo sueco de casos e controles não mostrou diferenças na avaliação de ansiedade entre obesos e normais (41). Outro estudo, australiano, mostrou a associação em meninas, ligando a ansiedade crônica à insatisfação com a imagem corporal, e esta, por sua vez, à obesidade (34). A associação pode se dever, também, à causalidade reversa, já que a coleta desses dados também foi contemporânea à mensuração do peso e adiposidade, porém isso não explicaria as diferenças entre os sexos.

Neste estudo, 10,3\% dos adolescentes $(13,7 \%$ dos meninos e $6,8 \%$ das meninas) relataram fumar pelo menos um cigarro por dia. Não houve associação entre a obesidade e o fumo. Resultados semelhantes foram encontrados em um estudo norte-americano (35).

A associação entre o consumo de gorduras e a obesidade é controversa $(32,36,37)$. O presente estudo utilizou, por facilidade logística, o inventário de Block (25), o qual não avalia o consumo calórico tão detalhadamente quanto os diários de consumo, cuja aplicação é mais complexa. Apesar da ausência de associação com obesidade, foi identificado um consumo elevado de gorduras tanto nos casos quanto nos controles.

Até o presente, não há instrumentos validados para medir a atividade física em adolescentes no Brasil. As propostas atuais de mensuração de atividade física em adolescentes em outros países $(38,39)$ têm validade apenas aceitável nas próprias populações onde foram desenvolvidas, além de não es- 
tarem disponíveis à época do estudo. O questionário utilizado na presente pesquisa foi extremamente detalhado, mas ainda assim não foi possível encontrar qualquer diferença entre obesos e não obesos. A literatura é controversa sobre a associação entre padrões de atividade física e a obesidade na adolescência. Um estudo sueco de casos e controles mostrou que adolescentes obesos são menos ativos fisicamente que os adolescentes de peso normal (40). Entretanto, um outro estudo de casos e controles realizado no mesmo país, comparando grupos de escolares com médias de diferença de peso de cerca de $40 \mathrm{~kg}$, não mostrou diferenças quanto ao tipo e duração das atividades físicas (41). Um estudo transversal em adolescentes escolares no Rio de Janeiro tampouco encontrou associação entre o sobrepeso e a atividade física (32). Cabe ainda notar, no presente estudo, que os níveis médios de atividade física relatados pelos adolescentes foram bastante elevados, e superiores à recomendação da OMS para atividade física em adultos (27).

Tanto para as atividades físicas quanto para o relato de consumo de gorduras, as informações coletadas foram contemporâneas à medida da obesidade. Assim, é possível que tenham sido afetadas por viés de informação, pois adolescentes obesos podem exagerar sua atividade física ou subestimar seu consumo de gorduras. Há também a possibilidade de causalidade reversa: adolescentes obesos podem ter aumentado sua atividade física ou reduzido o consumo de gorduras com o objetivo de perder peso.

No estudo do Rio de Janeiro, o tempo despendido assistindo à televisão e jogando videogames mostrou associação com sobrepeso em meninos, enquanto em nosso estudo a associação com a obesidade ocorreu no sexo feminino. Um estudo nacional americano, na faixa etária de 8 a 16 anos, mostrou maior adiposidade entre meninos e meninas que assistiam à televisão por mais de 4 horas diárias, quando comparados aos que assistiam à televisão por menos de 2 horas (42).

Dois aspectos metodológicos merecem especial atenção. Os presentes resultados confirmam a necessidade de estratificar todas as análises por sexo, uma vez que os fatores de risco para os meninos diferem substancialmente dos fatores observados entre as meninas. Em segundo lugar, as análises de determinantes contemporâneos devem ser ajustadas para fatores de confusão relativos à vida precoce dos adolescentes, pois muitas das associações brutas com fatores atuais desapareceram após esse tipo de ajuste.

O problema da determinação da obesidade na adolescência e a definição de seu papel na causalidade de morbidades futuras permanecem, ainda, um desafio. Nos últimos anos, tem aumentado o conhecimento sobre os fatores de risco em fases precoces da vida, paralelamente ao estudo de fatores contemporâneos, mais evidentes, mas não menos controversos. Os presentes resultados, assim como os achados deste estudo publicados anteriormente (9), sugerem que os fatores precoces têm um papel fundamental. A obesidade é um problema construído ao longo da vida, programado em sua fase precoce, e alimentado ao longo do tempo. É, provável, ainda, que uma parcela significativa de determinantes dessa morbidade ainda esteja por ser descoberta.

\section{REFERÊNCIAS}

1. World Health Organization. Obesity: preventing and managing the global epidemic. Genebra: World Health Organization; 1998.

2. Thompson D, Wolf AM. The medical-care cost burden of obesity. Obes Rev. 2001;2(3): 189-197.

3. Wang G, Dietz WH. Economic burden of obesity in youths aged 6 to 17 years: 1979-1999. Pediatrics. 2002;109(5):e81. Disponível em: http://www.pediatrics.org/cgi/content/full/ 109/5/e81. Acessado em agosto de 2004.

4. Centers for Disease Control and Prevention. National Center for Chronic Disease Prevention and Health Promotion. Defining overweight and obesity. Disponível em: http:// www.cdc.gov/nccdphp/dnpa/obesity/ defining.htm. Acessado em agosto de 2004.

5. Monteiro CA, Benício MH D'A, Conde WL, Popkin BM. Shifting obesity trends in Brazil. Eur J Clin Nutr. 2000;54(4):342-6.

6. Gigante DP, Barros FC, Post CLA, Olinto MTA. Prevalência de obesidade em adultos e seus fatores de risco. Rev Saude Publica. 1997; 31(3):236-46.

7. Ogden CL, Flegal KM, Carrol MD, Johnson CL. Prevalence and trends in overweight among US children and adolescents, 19992000. JAMA. 2002;288(14):1728-32.

8. Neutzling MB, Taddei JAAC, Rodrigues EM, Sigulem DM. Overweight and obesity in Brazilian adolescents. Int J Obes Relat Metab. Disord 2000;24(7):869-74.

9. Monteiro POA, Victora CG, Barros FC, Monteiro LMA. Birth size, early childhood growth, and adolescent obesity in a Brazilian birth cohort. Int J Obes Relat Metab Disord. 2003; 27(10)1274-82.

10. World Health Organization. Physical status: the use and interpretation of anthropometry. WHO: Genebra; 1995. (Technical Report series 854).

11. Smoak CG, Burke GL, Webber LS, Harsha DW, Srinivasan SR, Berenson GS. Relation of obesity to clustering of cardiovascular disease risk factors in children and young adults. The Bogalusa Heart Study. Am J Epidemiol. 1987; 125(3):364-72.

12. Goran MI, Ball GD, Cruz ML. Obesity and risk of type 2 diabetes and cardiovascular disease in children and adolescents. J Clin Endocrinol Metab. 2003;88(4):1417-27.
13. Strauss RS. Childhood obesity and selfesteem. Pediatrics. 2000;105(1):e15. Disponível em: http://www.pediatrics.org/cgi/ content/full/105/1/e15. Acessado em agosto de 2004.

14. Must A. Morbidity and mortality associated with elevated body weight in children and adolescents. Am J Clin Nutr. 1996;63(suppl): 445-7.

15. Must A, Jacques PF, Dallal GE, Bajema CJ, Dietz WH. Long-term morbidity and mortality of overweight adolescents. N Engl J Med. 1992;327(19):1350-5.

16. Gortmaker SL, Must A, Perrin JM, Sobol AM, Dietz WH. Social and economic consequences of overweight in adolescence and young adulthood. N Engl J Med. 1993;329(14): 1008-12.

17. Ebbeling CB, Pawlak DB, Ludwig DS. Childhood obesity: public-health crisis, common sense cure. Lancet. 2002;360(9331):473-82.

18. Stettler N, Bovet $P$, Shamlaye H, Zemel BS, Stallings VA, Paccaud F. Prevalence and risk factors for overweight and obesity in children from Seychelles, a country in a rapid transi- 
tion: the importance of early growth. Int J Obes Relat Metab Disord. 2002;26(2):214-9.

19. Parsons TJ, Power C, Logan S, Summerbell CD. Childhood predictors of adult obesity: a systematic review. Int J Obes. 1999;23(Suppl 8):1-107.

20. Victora CG, Barros FC, Lima RC, Behague DP, Gonçalves H, Horta BL, et al. The Pelotas (Brazil) Birth Cohort Study, 1982-2001. Cad Saude Publica. 2003;19(5):1241-56.

21. Monteiro POA, Victora CG, Barros FC, Tomasi E. Diagnóstico de sobrepeso em adolescentes: estudo do desempenho de diferentes critérios para o índice de massa corporal. Rev Saude Publica. 2000;34(5):506-13.

22. Harrison GG, Buskirk ER, Carter JEL, Johnston FE, Lohman TG, Pollock ML, et al. Skinfold thicknesses and measurement technique. Em: Lohmann TG, Roche AF, Martorell R, eds. Anthropometric standardization reference manual. Champaign, IL: Human Kinetics Books; 1988. Pp. 55-80.

23. Biaggio AMB, Natalício L. Manual para o Inventário de Ansiedade Traço-Estado (IDATE). Rio de Janeiro: Centro Editor de Psicologia Aplicada (CEPA); 1979.

24. Gorenstein C, Andrade L. Validation of a Portuguese version of the Beck Depression Inventory and the State-Trait Anxiety Inventory in Brazilian subjects. Braz J Med Biol Res. 1996;29(4):453-7.

25. Block G, Clifford C, Naughton MD, Henderson M, McAdams M. A brief dietary screen for high fat intake. J Nutr Educ. 1989;21(5): 199-207.

26. Ainsworth BE, Haskell WL, Leon AS, Jacobs DR Jr, Montoye HJ, Sallis JF, et al. Compendium of physical activities: classification of energy costs of human physical activities. Med Sci Sports Exerc. 1993;25(1):71-80.
27. Pate RR, Pratt M, Blair SN, Haskell WL, Macera CA, Bouchard C, et al. Physical activity and public health. A recommendation from the Centers for Disease Control and Prevention and the American College of Sports Medicine. JAMA. 1995;273(5):402-7.

28. Victora CG, Huttly SR, Fuchs SC, Olinto MTA. The role of conceptual frameworks in epidemiological analysis: a hierarchical approach. Int J Epidemiol. 1997;26(1):224- 7.

29. Mickey RM, Greenland S. The impact of confounder selection criteria on effect estimation. Am J Epidemiol. 1989;129(1):125-37.

30. De Spiegelaere M, Dramaix M, Hennart P. The influence of socioeconomic status on the incidence and evolution of obesity during early adolescence. Int J Obes Relat Metab Disord. 1998;22(3):268-74.

31. Laitinen J, Power C, Järvelin M-R. Family social class, maternal body mass index, childhood body mass index, and age at menarche as predictors of adult obesity. Am J Clin Nutr. 2001;74(3):287-94

32. Fonseca VM, Sichieri R, Veiga GV. Fatores associados à obesidade em adolescentes. Rev Saude Publica. 1998;32(6):541-9.

33. Whitaker RC, Wright JA, Pepe MS, Seidel KD, Dietz WH. Predicting obesity in young adulthood from childhood and parental obesity. N Engl J Med. 1997;337(13):869-73.

34. Kostanski M, Gullone E. Adolescent body image dissatisfaction: relationship with selfesteem, anxiety, and depression controlling for body mass. J Child Psychol Psychiat. 1998; 39(2):255-62.

35. Strauss RS, Mir HM. Smoking and weight loss attempts in overweight and normal-weight adolescents. J Obes Relat Metab Disord. 2001; 25(9):1381-5.
36. Magarey AM, Daniels LA, Boulton TJ, Cockington RA. Does fat intake predict adiposity in healthy children and adolescents aged 2-15y? A longitudinal analysis. Eur J Clin Nutr. 2002;56(10):1046-7.

37. Gillis LJ, Kennedy LC, Gillis AM, Bar-Or O. Relationship between juvenile obesity, dietary energy and fat intake and physical activity. Int J Obes Relat Metab Disord. 2002;26(4): 458-63.

38. Booth ML, Okely AD, Chey T, Bauman A. The reliability and validity of the physical activity questions in the WHO health behaviour in schoolchildren (HBSC) survey: a population study. Br J Sports Med. 2001;35(4):263-7.

39. Booth ML, Okely AD, Chey TN, Bauman A. The reliability and validity of the Adolescent Physical Activity Recall Questionnaire. Med Sci Sports Exerc. 2002;34(12):1986-95.

40. Ekelund U, Aman J, Yngve A, Renman C, Westerterp K, Sjöström M. Physical activity but not energy expenditure is reduced in obese adolescents: a case-control study. Am J Clin Nutr. 2002;76(5):935-41.

41. Renman C, Engström I, Silfverdal S-A, Aman J. Mental health and psychosocial characteristics in adolescent obesity: a population-based case-control study. Acta Paediatr. 1999;88(9): 998-1003.

42. Andersen RE, Crespo CI, Bartlett SJ, Cheskin LJ, Pratt M. Relationship of physical activity and television watching with body weight and level of fatness among children: results from the Third National Health and nutrition Examination Survey. JAMA. 1998;279(12):938-42.

Manuscrito recebido em 10 de novembro de 2003. Aceito em versão revisada em 3 de junho de 2004.
ABSTRACT

\section{Social, familial, and behavioral risk factors for obesity in adolescents}

Objective. To examine the impact of different social, familial, and behavioral factors on the risk of developing obesity in adolescents.

Methods. We performed a case-control study nested in a population-based cohort that was followed from birth in 1982. Cases were adolescents with obesity, as defined by the World Health Organization. Controls came from a randomly chosen sample of members of the cohort, examined in 1997 and 1998. Information concerning risk factors was obtained from cohort records that were collected at different ages. Multivariate analysis was carried out using logistic regression.

Results. Risk factors varied according to sex. Among boys, a family income at birth above one minimum wage was associated with a six-fold increase in obesity, and the presence of trait anxiety in adolescence with a four-fold increase. In both sexes, a oneunit increase in pre-pregnancy maternal body mass index was associated with a $10 \%$ increase in obesity. Smoking, fat consumption, time spent watching television or performing physical activity, and concurrent maternal weight were not associated with obesity.

Conclusions. Our results show that it is important to stratify data from obesity studies according to sex. In addition, early-life factors were more strongly associated with obesity than factors present during adolescence. However, the possibility of reporting bias cannot be ruled out, especially in terms of the information provided on diet and physical exercise. Whenever possible, the study of concurrent risk factors for obesity in adolescence should take into account the confounding effect of early-life factors. 